\title{
Trichophyton violaceum
}

National Cancer Institute

\section{Source}

National Cancer Institute. Trichophyton violaceum. NCI Thesaurus. Code C127759.

A species of anthropophilic fungi in the phylum Ascomycota with a worldwide distribution. This species causes inflammatory, or chronic non-inflammatory, finely scaling lesions of skin, nails, beard and scalp, producing black dot tinea capitis. 